\title{
LMA with positive pressure ventilation is safe!
}

\author{
Nicole Riem ${ }^{1}$, Sylvain Boet ${ }^{1}$, Laurent Tritsch $^{2}$, and Dylan Bould ${ }^{3}$ \\ ${ }^{1}$ Department of Anesthesia, St Michael's Hospital, University of Toronto, Toronto, Canada, ${ }^{2}$ Service Départemental d'Incendie et de \\ Secours (SDIS) du Bas-Rhin, 67000 Strasbourg, France, ${ }^{3}$ Children's Hospital of Eastern Ontario, Ontario, Canada
}

\section{Implication statement}

The purpose of this letter is to address two common techniques of ventilation with the LMA and briefly summarize evidence in literature as an answer to a recent publication cited in our correspondence. We believe the topic to be of interest for Anesthesiologists.

\section{Correspondence}

The laryngeal mask airway (LMA) has been found to be safe for airway management during general anaesthesia over the past 20 years. The LMA has a number of potential advantages over the endotracheal tube (ETT) including efficiency and hemodynamic stability during induction and emergence as well as improved recovery profile. It also requires only short training to learn insertion of a LMA. Also, the LMA has become one of the essential tools in management of the difficult airway. In spite of growing evidence it is still a controversial issue whether the LMA can be used with patients breathing spontaneously or with positive pressure ventilation (PPV). In UK and North America, spontaneous breathing is very common while in Europe patients with LMA seem to be routinely ventilated with positive pressure. Is there any evidence to support one technique more than the other or does choice rely only on personal preferences?

A common reason why anesthesiologists prefer to use the LMA with spontaneous breathing patients is it allows titration of analgesia according to the respiratory rate of the patient. With increasing experience of the anesthesiologist, the amount of used anesthetic drugs may be reduced to the minimum. The risk of side effects on pulmonary and cardio vascular functions from anesthetics may therefore be minimized. This allows the use of the LMA even in seriously ill patients [1].

The combination of regional technique and general anesthesia with a LMA in spontaneous mode is elegant and can be safely used for long procedures.

A very common reason to use the LMA in spontaneous breathing patients is prevention of gastric insufflations caused by high airway pressures, light anesthesia or a misplaced LMA. Misconceptions in relation to the use of LMA with PPV make anesthesia practitioners reluctant to choose the LMA over ETT. It stems from the belief that there is increased incidence of gastric insufflations with mechanical ventilation leading to increased risk to pulmonary aspiration of gastric contents. In addition, this risk is believed to increase more when muscle relaxation is used.

A recently published retrospective analysis of prospectively collected information on a large series of more than 65000 procedures confirms an incidence of aspiration comparable to previous studies [2]. The authors found that aspiration was as common when a tracheal tube was used (adjusted odds ratio of 1.06 [95\% CI 8.6-108.9]). In addition, similar patient outcomes were also found with patients spontaneously breathing with a LMA device and with non-paralyzed patients with PPV [3]. But even after years of controversy we are still struggling with the limitations of observational data. We do not know many important baseline risk factors for aspiration in each group of this large series that might allow us to truly determine the relative risk of this event for LMA versus a tracheal tube.

Corresponding author: Nicole Riem, M.D., Department of Anesthesia, St Michael's Hospital, University of Toronto, 30 Bond Street, Toronto, Ontario, M5B 1W8, Canada. Tel: 1-416-864-5071, Fax: 1-416-864-6014, E-mail: nriem@aol.com

(c) This is an open-access article distributed under the terms of the Creative Commons Attribution Non-Commercial License (http:// creativecommons.org/licenses/by-nc/3.0/), which permits unrestricted non-commercial use, distribution, and reproduction in any medium, provided the original work is properly cited. 
A potential important element to determine the safety of LMA with PPV might be the airway resistance and compliance. Devitt and his colleagues showed that LMA can be satisfactorily used for PPV in patients with normal airway resistance and compliance who do not require high insufflations pressures (> 17-20 $\mathrm{cmH}_{2} \mathrm{O}$ ) to produce normal tidal volumes [4]. Keller and his co-workers also concluded that gastroesophageal insufflations is no concern when ventilation pressures are kept lower than $20 \mathrm{cmH}_{2} \mathrm{O}$ with or without muscle relaxants [5]. Moreover, considering the quality of gas exchange, LMA with pressure support ventilation and PEEP of $5 \mathrm{cmH}_{2} \mathrm{O}$ provides more effective gas exchange than does spontaneous breathing with continuous positive airway pressure (CPAP) [6].

In summary, accepting contra indications of the LMA, PPV is strongly supported by all the published data as a safe practice.

\section{References}

1. Keller C, Brimacombe J. Spontaneous versus controlled respiration with the laryngeal mask. A review. Anaesthesist 2001; 50: 187-91.

2. Bernardini A, Natalini G. Risk of pulmonary aspiration with laryngeal mask airway and tracheal tube: analysis on 65712 procedures with positive pressure ventilation. Anaesthesia 2009; 64: 1289-94.

3. Keller C, Sparr HJ, Luger TJ, Brimacombe J. Patient outcomes with positive pressure versus spontaneous ventilation in non-paralysed adults with the laryngeal mask. Can J Anaesth 1998; 45: 564-7.

4. Devitt JH, Wenstone R, Noel AG, O'Donnell MP. The laryngeal mask airway and positive-pressure ventilation. Anesthesiology 1994; 80: 550-5.

5. Keller C, Sparr HJ, Luger TJ, Brimacombe J. Patient outcomes with positive pressure versus spontaneous ventilation in non-paralysed adults with the laryngeal mask. Can J Anaesth 1998; 45: 564-7.

6. Brimacombe J, Keller C, Hörmann C. Pressure support ventilation versus continuous positive airway pressure with the laryngeal mask airway: a randomized crossover study of anesthetized adult patients. Anesthesiology 2000; 92: 1621-3. 